\title{
Hyperbolic Conservation Laws with Relaxation $\star$
}

\author{
Tai-Ping Liu \\ Department of Mathematics, University of Maryland, College Park, MD 20742, USA
}

\begin{abstract}
The effect of relaxation is important in many physical situations. It is present in the kinetic theory of gases, elasticity with memory, gas flow with thermo-non-equilibrium, water waves, etc. The governing equations often take the form of hyperbolic conservation laws with lower-order terms. In this article, we present and analyze a simple model of hyperbolic conservation laws with relaxation effects. Dynamic subcharacteristics governing the propagation of disturbances over strong wave forms are identified. Stability criteria for diffusion waves, expansion waves and traveling waves are found and justified nonlinearly. Time-asymptotic expansion and the energy method are used in the analysis. For dissipative waves, the expansion is similar in spirit to the Chapman-Enskog expansion in the kinetic theory. For shock waves, however, a different approach is needed.
\end{abstract}

\section{Introduction}

The phenomenon of relaxation is important in many physical situations. In the kinetic theory of monatomic gases, when an equilibrium state is perturbed, it gradually relaxes to the equilibrium state with Maxwellian velocity distribution. In the continuum theory of nonmonatomic gases, there are other modes of internal energy besides the translated one, and when the gas is perturbed, the translational energy adjusts to its equilibrium value quickly. Other modes relax to their equilibrium values through collision of gas particles. The time scale for such a relaxation process may not be short and the phenomenon of thermononequilibrium becomes important. In this case, the compressible Euler equations should be supplemented by a rate equation governing the nonequilibrium mode of the internal energy. For elastic material with memory, the stress depends on the past history of the strain, and a perturbation of a constant state relaxes to the state satisfying the equilibrium elastic stress-strain relation. For a river flow to be in

* The paper was written at Mittag-Leffler Institute; the author wants to thank the Institute for the visiting position in 1986. This work was supported in part by an NSF grant 
equilibrium, the gravitational force and the frictional force with the riverbed are in balance, and the water flows with constant speed depending on the depth of the river. Other relaxation phenomena occur in MHD, traffic flows, etc. The process of relaxation to equilibrium states can be studied with the aid of the time-asymptotic expansion in deriving a simplified system. The expansion is valid only in the absence of strong permanent wave forms. For strong permanent waves such as traveling waves, certain dynamic subcharacteristics, different from the equilibrium subcharacteristics obtained through the asymptotic expansion, become the basic element in governing the propagation of disturbances. The purpose of the present article is to present these ideas and their analytical justification through a simple model. Subsequent studies will deal with other physical models.

Consider the following system of two quasilinear hyperbolic equations:

$$
\begin{gathered}
\frac{\partial u}{\partial t}+\frac{\partial f(u, v)}{\partial x}=0, \\
\frac{\partial v}{\partial t}+\frac{\partial g(u, v)}{\partial x}=h(u, v), \quad t \geqq 0, \quad-\infty<x<\infty .
\end{gathered}
$$

The first equation represents a conservation law for $u$ and the second equation a rate equation for $v$. The term $h(u, v)$ acts as a source (or sink) when $v$ is less (or larger) than its equilibrium value $v(u)$, which is a given function of $u$. Often $h(u, v)$ assumes the following form:

$$
h(u, v)=\frac{v_{*}(u)-v}{\tau(u)}
$$

for some positive function $\tau(u)$, the relaxation time. We make the following general assumption:

$$
\frac{\partial h(u, v)}{\partial v}<0, \quad h\left(u, v_{*}(u)\right)=0
$$

for all $(u, v)$ under consideration.

When the solution is close to equilibrium, one often ignores the rate equation and replaces the conservation law by the equilibrium equation:

$$
\frac{\partial u}{\partial t}+\frac{\partial f_{*}(u)}{\partial x}=0, \quad f_{*}(u) \equiv f\left(u, v_{*}(u)\right)
$$

The system (1.1) is assumed to be strictly hyperbolic with characteristics $\lambda_{1}(u, v)$ $<\lambda_{2}(u, v)$

$$
\begin{gathered}
\frac{\partial(f, g)}{\partial(u, v)} r_{i}=\lambda_{i} r_{i}, \quad i=1,2 \\
\lambda_{2}^{\prime}(u, v) \equiv 1 / 2\left(\frac{\partial f}{\partial u}+\frac{\partial g}{\partial v}\right) \mp\left(\left(\frac{\partial f}{\partial u}-\frac{\partial g}{\partial v}\right)^{2}+4 \frac{\partial f}{\partial v} \frac{\partial g}{\partial u}\right)^{1 / 2} .
\end{gathered}
$$

The equilibrium characteristic for (1.3) is:

$$
\lambda_{*}(u) \equiv f_{*}^{\prime}(u) \text {. }
$$

For a finite perturbation of an equilibrium state $\left(u_{0}, v_{*}\left(u_{0}\right)\right)$, the solution eventually relaxes to $\left(u_{0}, v_{*}\left(u_{0}\right)\right)$. In such a process the equilibrium characteristic 
$\lambda_{*}$ plays the basic role in the propagation of the disturbance. However, the process is not approximated accurately by the equilibrium equation (1.3). Instead, waves diffuse and are approximated accurately by a viscous conservation law of the form

$$
\frac{\partial u}{\partial t}+\frac{\partial f_{*}(u)}{\partial x}=\frac{\partial}{\partial x}\left(\beta(u) \frac{\partial u}{\partial x}\right) .
$$

In Sect. 2, we derive such an equation for diffusion waves as well as for rarefaction waves. The derivation differs from previous ones in that it is completely nonlinear and is based on the time-asymptotic expansion rather than the asymptotic expansion with small relaxation time, cf. [7]. The validity of our expansion can be justified. Indeed, in Sect. 3 we show that under the assumption that $\lambda_{*}$ is a subcharacteristic, $\lambda_{1}<\lambda_{*}<\lambda_{2}$, diffusion waves and expansion waves for (1.1) are nonlinearly stable and are accurately approximated time-asymptotically by (1.6).

System (1.1) admits traveling waves $(u, v)(x, t)=(\varphi, \psi)(x-\sigma t), \sigma$ the speed of the wave. It turns out the traveling waves for (1.1) are directly related to the shock waves for (1.3). For each admissible shock wave of (1.3) there exists a traveling wave for (1.1) with the property that the speed $\sigma$ lies between $\lambda_{1}$ and $\lambda_{2}$. This and other properties are studied in Sect. 4. A traveling wave is a permanent wave form. The nature of its stability differs markedly from that of a rarefaction wave. In Sect. 5, we show that a traveling wave satisfying $\lambda_{1}<\sigma<\lambda_{2}$ is non-linearly stable. However, instead of the equilibrium characteristic speed $\lambda_{*}$, a perturbation is governed by a certain dynamic subcharacteristic,

$$
\mu \equiv \frac{\partial f}{\partial u}-\frac{\partial f}{\partial v} \frac{\partial h}{\partial u}\left(\frac{\partial h}{\partial v}\right)^{-1} .
$$

The stability is partly the consequence of the compressibility of the traveling wave with respect to $\mu$. The dynamic speed $\mu$ is close to the equilibrium speed $\lambda_{*}$ for weak waves, but in general they are different.

Finally in Sect. 6 we study wave patterns which represent time-asymptotic states of solutions of (1.1) with different equilibrium states at $x= \pm \infty$. Such a wave pattern consists of traveling waves and expansion waves. Weak traveling waves are smooth. Strong traveling waves may contain discontinuity waves of the two conservation laws

$$
\frac{\partial u}{\partial t}+\frac{\partial f(u, v)}{\partial x}=0, \quad \frac{\partial v}{\partial t}+\frac{\partial g(u, v)}{\partial x}=0 .
$$

We use the theory of [5] to study such discontinuity waves. The expansion waves are based on the equilibrium equation (1.3) and are not exact but time-asymptotic solutions of (1.1). A new procedure is introduced for the construction of wave patterns.

Many physical examples are of the form (1.1), cf. [4, 7]. For more complicated physical models, $u \in \mathbb{R}^{n}, n>1$, there is more than one subcharacteristic speed and more than one mode may eventually survive. In this case, to study the stability of waves one needs to decompose the solution into several elementary waves and use a certain hyperbolic technique in addition to the energy method employed here, 
[6]. The study of stability of traveling waves for a model in the kinetic theory is being carried out, [1]. It would be interesting to generalize the theory to other physical models, $[2,3,7]$.

\section{Time Asymptotic Expansion}

In this section we perform the time-asymptotic expansion for (1.1) and assess its validity. The first-order expansion is the equilibrium equation (1.3). For (1.3) we have

$$
\frac{\partial u}{\partial t}+\lambda_{*}(u) \frac{\partial u}{\partial x}=0 \text {. }
$$

For second-order approximation we set

$$
v=v_{*}(u)+v_{1} .
$$

Instead of assuming that $v_{*}(u)$ satisfies $(1.3)$ we require that $v_{*}(u)+v_{1}$ satisfies a new equation. The equilibrium equation (1.3) is used to determine the primary direction of wave propagation.

$$
\frac{\partial}{\partial t}+\lambda_{*}(u) \frac{\partial}{\partial x} \cong 0 .
$$

In deriving (1.3), the rate equation in (1.1) is ignored. We now use the rate equation to find $v_{1}$ :

$$
\frac{\partial}{\partial t}\left(v_{*}(u)+v_{1}\right)+\frac{\partial}{\partial x}\left(g\left(u, v_{*}(u)+v_{1}\right)\right) \cong h(u, v)-h\left(u, v_{*}(u)\right),
$$

where we have used (1.2). Since $v_{1}$ is the second-order correction, the above is simplified into (2.1),

$$
\frac{\partial v_{*}(u)}{\partial t}+\frac{\partial g\left(u, v_{*}(u)\right)}{\partial x} \cong \frac{\partial h\left(u, v_{*}(u)\right)}{\partial v} v_{1} .
$$

Finally from (2.2) we arrive at

$$
v_{1} \cong\left(\frac{\partial h\left(u, v_{*}(u)\right)}{\partial v}\right)^{-1}\left(-v_{*}^{1}(u) \lambda_{*}(u) \frac{\partial u}{\partial x}+\frac{g\left(u, v_{*}(u)\right)}{\partial x}\right),
$$

with the simplified notations

$$
h_{*}(u) \equiv h\left(u, v_{*}(u)\right), \quad g_{*}(u) \equiv g\left(u, v_{*}(u)\right), \quad \frac{\partial h_{*}}{\partial v}(u) \equiv \frac{\partial h}{\partial v}\left(u, v_{*}(u)\right), \quad \text { etc. }
$$

The value for $v_{1}$ is finally set to be

$$
v_{1} \equiv\left(\frac{\partial h_{*}}{\partial v}\right)^{-1}\left(-v_{*}^{1}(u) \lambda_{*}(u)+\frac{\partial g_{*}}{\partial u}+\frac{\partial g_{*}}{\partial v} v_{*}^{1}(u)\right) \frac{\partial u}{\partial x} \equiv \alpha(u) \frac{\partial u}{\partial x} .
$$

Plug (2.1) and (2.3) into the conservation law in (1.1) and we get

$$
\frac{\partial u}{\partial t}+\frac{\partial}{\partial x} f\left(u, v_{*}(u)-\alpha(u) \frac{\partial u}{\partial x}\right)=0 .
$$


This equation is approximated by

$$
\frac{\partial u}{\partial t}+\frac{\partial f_{*}(u)}{\partial x}=\frac{\partial}{\partial x}\left(\frac{\partial f_{*}}{\partial v}(u) \alpha(u) \frac{\partial u}{\partial x}\right),
$$

or

$$
\begin{gathered}
\frac{\partial u}{\partial t}+\frac{\partial f_{*}(u)}{\partial x}=\frac{\partial}{\partial x}\left(\beta(u) \frac{\partial u}{\partial x}\right) \\
\beta(u) \equiv \frac{\partial f_{*}}{\partial v}(u)\left(-v_{*}^{\prime}(u) \lambda_{*}(u)+\frac{\partial g_{*}}{\partial u}(u)+\frac{\partial g_{*}}{\partial v}(u) v_{*}^{\prime}(u)\right) .
\end{gathered}
$$

The above procedure is of the same spirit as the Chapman-Enskog expansion for the Boltzmann equation, [2]. For the Hilbert expansion one would require $v_{*}(u)$ in (2.1) to satisfy (1.3) and $v_{1}$ to satisfy an inhomogeneous conservation law. Such an expansion cannot be justified the way Chapman-Enskog expansion can. One usually justified these expansions for small mean free path. In the present situation, write

$$
h(u, v)=\frac{v_{*}(u)-v}{\tau(u)},
$$

where $\tau(u)$ is the relaxation time. Equations (1.3) and (2.4) may be viewed as the asymptotic expansions for small $\tau(u)$. Since our interest is on the behavior of solutions for (1.1) with finite relaxation time, we will assess the validity of (2.4) time asymptotically. Such an analysis will be useful for the study of large-time behavior of solutions (1.1). Equation (2.4) was derived based on the principle that

$$
u \gg \nabla u \gg \nabla^{2} u .
$$

This holds for diffusion waves and expansion waves of (2.4) as $t \rightarrow \infty$. In other words, given a diffusion wave or an expansion wave $u(x, t)$ of $(2.4)$, set $v(x, t)$ according to $(2.2)$ and $(2.3)$; we expect $(u, v)(x, t)$ to represent a time-asymptotic state of the original system (1.1). The purpose of such a time-asymptotic expansion is to obtain the simplified system, (2.4), which is easier to analyze and yet represents an important phase, the large-time behavior, of the original system, (1.1).

The derived equation (2.4) is well-posed only if the diffusion coefficient $\beta(u)$ is positive. From (1.4) and (1.5) it follows that

$$
\beta(u)=-\left(\lambda_{*}-\lambda_{1}\right)\left(\lambda_{*}-\lambda_{2}\right) .
$$

Thus the stability criterion becomes

$$
\lambda_{1}<\lambda_{*}<\lambda_{2},
$$

that is, $\lambda_{*}$ is subcharacteristic.

For a perturbation of an equilibrium state $\left(u_{0}, v_{*}\left(u_{0}\right)\right),(2.2)-(2.4)$ govern the dissipation process as $t \rightarrow \infty$. The justification would involve the study of linearization of (1.1) around the nonlinear diffusion waves provided by (2.4). The time-asymptotic equivalence of (1.1) and (2.2)-(2.4) would be both in $L_{1}(x)$ and $L_{\infty}(x)$ sense. On the other hand, for rarefaction waves, the stability is in a weaker sense and the equivalence is expected only in $L_{\infty}(x)$ sense. For $L_{\infty}$ equivalence, (2.4) 
may be replaced by the simpler equation (1.3). In the next section we study such an equivalence. One basic assumption we will make throughout is that the two equations in (1.1) are coupled. Precisely we assume that

$$
\frac{\partial f}{\partial v}(u, v) \neq 0 \quad \text { for all }(u, v) \text { under consideration. }
$$

\section{Nonlinear Stability of Rarefaction Waves}

When $f_{*}^{\prime \prime}(u) \neq 0$ and the initial data is such that $\lambda_{*}(x, 0)$ is a nondecreasing function of $x$ then the solution of the equilibrium equation (1.3) is an expansion (rarefaction) wave. Given any state $u_{-}$and $u_{+}, \lambda_{*}\left(u_{-}\right)<\lambda_{*}\left(u_{+}\right)$, we may construct a smooth expansion wave $\varphi(x, t)$ of $(1.3)$ with $\varphi( \pm \infty, t)=u_{ \pm}$by setting

$$
\varphi(x, 0)= \begin{cases}u & \text { for } x<-k \\ u_{0}(x) & \text { for }|x| \leqq k \\ u_{+} & \text {for } x>k\end{cases}
$$

where $k$ is a fixed positive constant and $u_{0}(x)$ any smooth monotone function. Suppose that $(u, v)(x, t)$ is any solution of (1.1) with equilibrium states $\left(u_{ \pm}, v_{ \pm}\right)$, $v_{ \pm}=v_{*}\left(u_{ \pm}\right)$, at $x= \pm \infty$. Our purpose of this section is to show that $(u, v)(x, t)$ $\rightarrow\left(\varphi, v_{*}(\varphi)\right)(x, t)$ as $t \rightarrow \infty$. Since a translation or dilation of a rarefaction wave is also a rarefaction, the convergence here is in the $L_{\infty}$ sense and not in $L_{1}$. This is the reason we do not use the more accurate approximation (2.2)-(2.4) but (1.3) in constructing the rarefaction wave $(\varphi, \psi) \equiv\left(\varphi, v_{*}(\varphi)\right)$. The rarefaction wave expands at the rate $t$ :

$$
\begin{aligned}
& \left(\left|D^{\alpha} \psi\right|+\left|D^{\alpha} \psi\right|\right)(x, t) \\
& \quad=\left\{\begin{array}{lll}
0 & \text { for } & |x|>m t+k \\
O(1) \varepsilon_{0} t^{-|\alpha|} & \text { for } & |x|<m t+k, \varepsilon_{0} \equiv\left|u_{+}-u_{-}\right|,
\end{array}\right.
\end{aligned}
$$

for some positive constant $m \equiv \max \left\{\left|f^{\prime}(u)\right|: u\right.$ between $u_{-}$and $\left.u_{+}\right\}$and any partial derivatives $D^{\alpha} \varphi$ of $\varphi$ with respect to $x$ and $t$. The first step is to assess the accuracy of $(\varphi \cdot \psi)$ as an approximate solution of (1.1). Since $\varphi$ solves (1.3) and $\psi=v_{*}(\varphi)$, we have

$$
\frac{\partial \varphi}{\partial t}+\frac{\partial f(\varphi, \psi)}{\partial x}=\frac{\partial \varphi}{\partial t}+\frac{\partial f_{*}(\varphi)}{\partial x}=0
$$

Since $\psi=v_{*}(\varphi), h(\varphi, \psi)=0$ and so from (3.2),

$$
\begin{gathered}
\frac{\partial \psi}{\partial t}+\frac{\partial g(\varphi, \psi)}{\partial x}=h(\varphi, \psi)+F, \\
D^{\alpha} F=O(1) \frac{\partial \varphi^{|\alpha|+1}}{\partial x^{|\alpha|+1}}=\left\{\begin{array}{lll}
0 & \text { for } & |x|>m t+K \\
O(1) \varepsilon_{0} t^{-1-|\alpha|} & \text { for } & |x| \leqq m t+k .
\end{array}\right.
\end{gathered}
$$

Consider a perturbation of $(\varphi, \psi)$ :

$$
(u, v)(x, t)=(z+\varphi, w+\psi)(x, t), \quad(z, w)( \pm \infty, 0)=0 .
$$


Theorem 3.1. Suppose that $\lambda_{*}$ is a subcharacteristic, $\lambda_{1}<\lambda_{*}<\lambda_{2}$, for all states under consideration, and that the rarefaction wave $(\varphi, \psi)$ and the perturbation $(\bar{u}, \bar{v})$ are weak, $\varepsilon(0) \ll 1$, where

$$
\varepsilon(t) \equiv\left|u_{+}-u_{-}\right|+\max _{x} \sum_{i=0}^{3}\left|\frac{\partial^{i}(z, w)}{\partial x^{i}}(x, t)\right|^{2} .
$$

Then the smooth solution $(u, v)(x, t)$ of (1.1) exists for all time and tends to the equilibrium rarefaction wave $(\varphi, \psi)$ as $t \rightarrow \infty$ :

$$
\lim _{t \rightarrow \infty} \sup _{-\infty \leqq x \leqq \infty}(|z(x, t)|+|w(x, t)|)=0 .
$$

Proof. Subtract (3.3) from (1.1) to obtain

$$
\begin{gathered}
\frac{\partial z}{\partial t}+\frac{\partial}{\partial x}(f(z+\varphi, w+\psi)-f(\varphi, \psi))=0, \\
\frac{\partial w}{\partial t}+\frac{\partial}{\partial x}(g(z+\varphi, w+\psi)-g(\varphi, \psi))=h(z+\varphi, w+\psi)-h(\varphi, \psi)-F .
\end{gathered}
$$

We have from (3.5), that

$$
\left(f_{v} w\right)_{x}=-z_{t}-\left(f_{u} z\right)_{x}-\left[f_{u}\right] z_{x}-\left[f_{v}\right] w_{x}-Q_{0}\left(f_{u}\right) \phi_{x}-Q_{0}\left(f_{v}\right) \psi_{x},
$$

and for $z$ and $w$ sufficiently small, cf. (2.8),

$$
w_{x}=\left(f_{v}+\left[f_{v}\right]\right)^{-1}\left(-z_{t}-f_{u} z_{x}-\left[f_{u}\right]\left(z_{x}+\phi_{x}\right)-\left[f_{v}\right] \psi_{x}\right) .
$$

Here and thereafter the functions $f_{u} \equiv \partial f / \partial u, g_{v} \equiv \partial g / \partial v$, etc., are evaluated at $(\varphi, \psi)$, and for any function $\beta$,

$$
\begin{gathered}
{[\beta]=\beta(z+\varphi, w+\psi)-\beta(\varphi, \psi)=O(1)(|z|+|w|),} \\
Q_{0}(\beta)=[\beta]-\beta_{u} z-\beta_{v} w=O(1)\left(z^{2}+w^{2}\right), \\
Q_{1}(\beta)=[\beta]-\beta_{u} z-\beta_{v} w-\beta_{u u} z^{2}-2 \beta_{u v} z w-\beta_{v v} w^{2}=O(1)\left(|z|^{3}+|w|^{3}\right) .
\end{gathered}
$$

Plug (3.6) into $h_{v}\left(-f_{v} h_{v}^{-1}(3.5)_{2}\right)_{x}$ to eliminate $w$ and obtain

$$
\begin{aligned}
z_{t t}+ & \left(\lambda_{1}^{+} \lambda_{2}\right) z_{x t}+\lambda_{1} \lambda_{2} z_{x x}-h_{v}\left(z_{t}+\left(\lambda_{*} z\right)_{x}\right) \\
= & -f_{v}\left(h_{u u} z z+h_{u v} z w+h_{v v} w w\right)_{x}+h_{v}\left(f_{u u} z^{2}+2 f_{u v} z w+f_{v v} w^{2}\right)_{x} \\
& +h_{v}\left(Q_{1}(f)\right)_{x}-f_{v}\left(Q_{0}\left(h_{v}\right)\left(z_{x}+\varphi_{x}\right)+Q_{0}\left(h_{u}\right)\left(w_{x}+\psi_{x}\right)\right) \\
& +\left(f_{v t} f_{v}^{-1}-\lambda_{0} f_{v x} f_{v}^{-1}-h_{v}\left(g_{v} h_{v}^{-1}\right)_{x}\right)\left(z_{t}-\lambda_{0} z_{x}\right) \\
& \left.-\left(f_{v}\left(f_{z} f_{v}^{-1}\right)_{t}-\lambda_{0} f_{v}\left(f_{u} f_{v}^{-1}\right)_{x}\right)+\left(f_{u} g_{v} h_{v}^{-1}\right)_{x}\right) z_{x} \\
& -f_{v} h_{v}^{-1} h_{v x}\left(w_{t}-\lambda_{0} w_{x}\right)-\left(f_{v}^{-1}\left[f_{u}\right]\left(z_{x}+\varphi_{x}\right)\right. \\
& \left.\left.\left.+f_{v}^{-1}\left[f_{v}\right]\left(w_{x}+\psi_{x}\right)\right)_{t}-\lambda_{0}\right) f_{v}^{-1}\left[f_{u}\right]\left(z_{x}+\varphi_{x}\right)+f_{v}^{-1}\left[f_{v}\right]\left(w_{x}+\psi_{x}\right)\right)_{x} \\
& -h_{v}\left(h_{v}^{-1} g_{v}\left[f_{u}\right]\left(z_{x}+\varphi_{x}\right)+h_{v}^{-1} g_{v}\left[f_{v}\right]\left(w_{x}+\psi_{x}\right)\right)_{x} \\
\equiv & \text { R.H.S. }
\end{aligned}
$$

In (3.9) the speed of propagation of the rarefaction wave $(\varphi, \psi)$ is small. This is achieved through the transformation $t \rightarrow t, x \rightarrow x-\lambda_{0} t, \lambda_{0}$ the average speed 
$1 / 2\left(\lambda_{*}\left(u_{-}\right)+\lambda_{*}\left(u_{+}\right)\right)$. Since $\left|u_{-}-u_{+}\right|$is small, we have from (3.5) and the structure of rarefaction waves that

$$
\begin{gathered}
\lambda_{1} \lambda_{2}<0, \\
\left|\phi_{t}\right|+\left|\psi_{t}\right| \ll\left|\phi_{x}\right|, \quad\left|\lambda_{*}\right| \ll 1 .
\end{gathered}
$$

Because the rarefaction wave is expansive, we have

$$
\lambda_{* x}>0, \quad C \lambda_{* x}>\left|\phi_{x}\right|
$$

for some constant $C>0$.

In the energy estimate that follows, we make the following a priori assumption:

$$
\varepsilon \equiv \max _{0 \leqq t \leqq \tau} \varepsilon(t) \ll 1
$$

Multiply (3.9) by $z$ and integrate over $0 \leqq t \leqq \tau$ and $-\infty<x<\infty$, and use (3.10)-(3.12) and (1.2) to obtain

$$
\begin{aligned}
\frac{1}{2} & \left.\int_{-\infty}^{\infty}\left(\left|h_{v}\right| z^{2}\right)(x, \tau) d x+\frac{1}{2} \int_{0}^{\tau} \int_{-\infty}^{\infty}\left(\left|h_{v} \lambda_{* x}\right|+h_{v t}+\lambda_{*} h_{v x}\right) z^{2}+\left|\lambda_{1} \lambda_{2}\right| z_{x}^{2}\right) d x d t \\
& =\frac{1}{2} \int_{-\infty}^{\infty}\left(\left(\left|h_{v}\right| z^{2}\right)(x, 0)+\left(z z_{t}(x, 0)-\left(z z_{t}(x, \tau)\right) d x+\int_{0}^{\tau} \int_{-\infty}^{\infty} \text { R.H.S. } d x d t\right.\right.
\end{aligned}
$$

We next estimate the integral of the R.H.S. From Schwartz inequality

$$
z^{4}(x)=\left(\int_{-\infty}^{x} 2 z z_{x} d_{x}\right)^{2} \leqq \int_{-\infty}^{\infty} z_{x}^{2} d x \int_{-\infty}^{\infty} z_{x}^{2} d x,
$$

and so

$$
\int_{0}^{\tau} \int_{-\infty}^{\infty} z^{6} d x d t \leqq\left(\max _{0 \leqq t \leqq \tau}\left(\int_{-\infty}^{\infty} z^{2}(x, t) d x\right)^{2}\right) \int_{0}^{\tau} \int_{-\infty}^{\infty} z_{x}^{2} d x d t \leqq \varepsilon \int_{0}^{\tau} \int_{-\infty}^{\infty} z_{x}^{2} d x d t .
$$

Thus

$$
\int_{0}^{\tau} \int_{-\infty}^{\infty}\left(z^{6}+w^{6}\right) d x d t \leqq \varepsilon \int_{0}^{\tau} \int_{-\infty}^{\infty}\left(z_{x}^{2}+w_{x}^{2}\right) d x d t
$$

For any given smooth function $\beta$ of $(\varphi, \psi)$, we have from (3.11) and (3.12) that

$$
\begin{aligned}
\int_{0}^{\tau} \int_{-\infty}^{\infty} \beta z^{2} z_{t} d x d t & =\frac{1}{3} \int_{-\infty}^{\infty}\left(\beta z^{3}\right)(x, \tau)-\left(\beta z^{3}\right)(x, 0) d x-\frac{1}{3} \int_{0}^{\tau} \int_{-\infty}^{\infty} \beta_{t} z^{3} d x d t \\
& =O(1) \varepsilon\left(\int_{-\infty}^{\infty}\left(z^{2}(x, \tau)+z^{2}(x, 0)\right) d x+\int_{0}^{\tau} \int_{-\infty}^{\infty} \lambda_{* x} z^{2} d x d t\right) .
\end{aligned}
$$

Similarly,

$$
\int_{0}^{\tau} \int_{-\infty}^{\infty} \beta z^{2} z_{t} d x d t=O(1) \varepsilon \int_{0}^{\tau} \int_{-\infty}^{\infty} \lambda_{* x} z^{2} d x d t
$$

From (3.5)

$$
w=h_{v}^{-1}\left(w_{t}+\left(g_{u} z\right)_{x}+\left(g_{v} w\right)_{x}+Q_{0}(g)_{x}-h_{u} z-Q_{0}(h)+F\right) .
$$


From (3.8), (3.11), (3.12), (3.17), and by parts,

$$
\begin{aligned}
\int_{0}^{\tau} \int_{-\infty}^{\infty} \beta z^{2} w_{t} d x d t= & O(1) \varepsilon\left(\int_{-\infty}^{\infty}\left(z^{2}(x, 0)+z^{2}(x, \tau)\right) d x+\int_{0}^{\tau} \int_{-\infty}^{\infty}\left(\lambda_{* x}\left(z^{2}+w^{2}\right)\right.\right. \\
& \left.\left.+|\nabla(x, w)|^{2}+\left(F_{x}\right)^{2}\left(\lambda_{* x}\right)^{-1}+z^{6}+w^{6}\right) d x d t\right),
\end{aligned}
$$

whence we have from $(3.3)_{3}$ and (3.15) that

$$
\begin{aligned}
\int_{0}^{\tau} \int_{-\infty}^{\infty} \beta z^{2} w_{t} d x d t= & O(1) \varepsilon\left(\int_{-\infty}^{\infty}\left(z^{2}(x, 0)+z^{2}(x, \tau)\right) d x+\int_{0}^{\tau} \int_{-\infty}^{\infty}\left(\lambda_{* x}\left(z^{2}+w^{2}\right)\right.\right. \\
& \left.\left.+|\nabla(z, w)|^{2}+\left|\lambda_{* x}\right|^{3}\right) d x d t\right) \equiv O(1) \varepsilon I
\end{aligned}
$$

Similarly we have

$$
\begin{aligned}
\left|\int_{0}^{\tau} \int_{-\infty}^{\infty} \beta z^{2} w_{x} d x d t\right| & +\left|\int_{0}^{\tau} \int_{-\infty}^{\infty} \beta z z_{x} w d x d t\right|+\left|\int_{0}^{\tau} \int_{-\infty}^{\infty} \beta z_{t} w^{2} d x d t\right|+\left|\int_{0}^{\tau} \int_{-\infty}^{\infty} \beta z w w_{t} d x d t\right| \\
& +\left|\int_{0}^{\tau} \int_{-\infty}^{\infty} \beta w^{2} z_{x} d x d t\right|+\left|\int_{0}^{\tau} \int_{-\infty}^{\infty} \beta z w w_{x} d x d t\right|=O(1) \varepsilon I
\end{aligned}
$$

The estimates (3.16) $)_{1}-(3.16)_{4}$ take care of the integral R.H.S. in (3.14). The second term on the left-hand side of (3.14) is treated by (3.11) and (3.12), and we conclude that

$$
\begin{aligned}
& \int_{-\infty}^{\infty} z^{2}(x, \tau) d x+\int_{0}^{\tau} \int_{-\infty}^{\infty}\left(\left|\lambda_{* x}\right| z^{2}+z_{x}^{2}\right) d x d t \\
& =O(1) \int_{-\infty}^{\infty} z^{2}(x, 0)+O(1) \int_{0}^{\tau} \int_{-\infty}^{\infty}\left(z_{t}^{2}+\varepsilon \lambda_{* x} w^{2}+\varepsilon|\nabla w|^{2}\right) d x d t,
\end{aligned}
$$

where we have used the hypothesis (3.13). Similar arguments yield the estimates for higher derivatives of $z$ by integrating (3.9) $z_{t},(3.9)_{x} z_{x},(3.9)_{t} z_{t t},(3.9)_{t} z_{t x},(3.9)_{x x} z_{x x}$, $(3.9)_{x x} z_{x x t},(3.9)_{t x} z_{t t x},(3.9)_{t t} z_{t t t}$, respectively,

$$
\begin{aligned}
& \int_{-\infty}^{\infty} z_{t}^{2}(x, \tau) d x+\int_{0}^{\tau} \int_{-\infty}^{\infty} z_{t}^{2} d x d t=O(1) \int_{-\infty}^{\infty} z_{t}^{2}(x, 0) d x \\
& +O(1) \int_{0}^{\tau} \int_{-\infty}^{\infty}\left(z_{x x}^{2}+\varepsilon\left(|\nabla, w|^{2}+\lambda_{* x}\left(z^{2}+w^{2}\right)+z_{t t}^{2}+z_{x t}^{2}+\left|\lambda_{* x}\right|^{3}\right)\right) d x d t \\
& \int_{-\infty}^{\infty} z_{x}^{2}(x, \tau) d x+\int_{0}^{\tau} \int_{-\infty}^{\infty} z_{x x}^{2} d x d t=O(1) \int_{-\infty}^{\infty} z_{x}^{2}(x, 0) d x \\
& +O(1) \int_{0}^{\tau} \int_{-\infty}^{\infty}\left(z_{x t}^{2}+\varepsilon|\nabla(z, w)|^{2}+\lambda_{* x}\left(z^{2}+w^{2}\right)+\left|\lambda_{* x}\right|^{3}\right) d x d t \\
& \int_{-\infty}^{\infty} z_{t t}^{2}(x, \tau) d x+\int_{0}^{\tau} \int_{-\infty}^{\infty} z_{t t}^{2} d x d t=O(1) \int_{-\infty}^{\infty}\left(z_{t t}^{2}(x, 0)+z_{x t}^{2}(x, \tau)+z_{x t}^{2}(x, 0)\right) d x \\
& +O(1) \int_{0}^{\tau} \int_{-\infty}^{\infty} \varepsilon\left(|\nabla(z, w)|^{2}+\lambda_{* x}\left(z^{2}+w^{2}\right)+\left|\nabla^{2}(z, w)\right|^{2}+z_{t t x}^{2}+z_{t t t}^{2}\right) d x d t
\end{aligned}
$$




$$
\begin{aligned}
& \int_{-\infty}^{\infty} z_{t x}^{2}(x, \tau) d x+\int_{0}^{\tau} \int_{-\infty}^{\infty} z_{t x}^{2} d x d t=O(1) \int_{-\infty}^{\infty}\left(z_{t x}^{2}(x, 0)+z_{x x}^{2}(x, \tau)+z_{x x}^{2}(x, 0)\right) d x \\
& +O(1) \int_{0}^{\tau} \int_{-\infty}^{\infty} \varepsilon\left(|\nabla(z, w)|^{2}+\lambda_{* x}\left(z^{2}+w^{2}\right)+\left|\nabla^{2}(z, w)\right|^{2}+\left|\lambda_{* x}\right|^{3}+z_{t x x}^{2}+z_{t t x}^{2}\right) d x d t,
\end{aligned}
$$

$$
\begin{aligned}
& \int_{-\infty}^{\infty} z_{x x}^{2}(x, \tau) d x+\int_{0}^{\tau} \int_{-\infty}^{\infty} z_{x x x}^{2} d x d t=O(1) \int_{-\infty}^{\infty}\left(z_{x x}^{2}(x, 0)+z_{x x t}^{2}(x, \tau)+z_{x x t}^{2}(x, 0)\right) d x \\
& +O(1) \int_{0}^{\tau} \int_{-\infty}^{\infty}\left(z_{x x t}^{2}+\varepsilon\left(|\nabla(z, w)|^{2}+\left|\nabla^{2}(z, w)\right|^{2}+\lambda_{* x}\left(z^{2}+w^{2}\right)+\left|\lambda_{* x}\right|^{3}+w_{x x x}^{2}\right)\right) d x d t,
\end{aligned}
$$

$$
\begin{aligned}
& \int_{-\infty}^{\infty}\left(z_{x x x}^{2}(x, \tau)+z_{x x t}^{2}(x, \tau)\right) d x+\int_{0}^{\tau} \int_{-\infty}^{\infty} z_{t x x}^{2} d x d t=O(1) \int_{-\infty}^{\infty}\left(z_{x x x}^{2}(x, 0)+z_{x x t}^{2}(x, \tau)\right) d x \\
& +O(1) \int_{0}^{\tau} \int_{-\infty}^{\infty} \varepsilon\left(|\nabla(z, w)|^{2}+\left|\nabla^{2}(z, w)\right|^{2}+\lambda_{* x}\left(z^{2}+w^{2}\right)+\left|\nabla^{3}(z, w)\right|^{2}+\left|\lambda_{* x}\right|^{3}\right) d x d t
\end{aligned}
$$

$$
\begin{aligned}
& \int_{-\infty}^{\infty}\left(z_{t t x}^{2}(x, \tau)+z_{t x x}^{2}(x, \tau)\right) d x+\int_{0}^{\tau} \int_{-\infty}^{\infty} z_{t t x}^{2} d x d t=O(1) \int_{-\infty}^{\infty}\left(z_{t t x}^{2}(x, 0)+z_{t x x}^{2}(x, \tau)\right) d x \\
& +O(1) \int_{0}^{\tau} \int_{-\infty}^{\infty} \varepsilon\left(|\nabla(z, w)|^{2}+\left|\nabla^{2}(z, w)\right|^{2}+\lambda_{* x}\left(z^{2}+w^{2}\right)+\left|\nabla^{3}(z, w)\right|^{2}+\left|\lambda_{* x}\right|^{3}\right) d x d t .
\end{aligned}
$$

We next turn to the estimates for $w$. Integrate $(3.5)_{2 t} w_{t},(3.5)_{2 t t} w_{t t}$, respectively, and use (1.2) to obtain

$$
\begin{aligned}
& \int_{\infty}^{\infty} w_{t}^{2}(x, \tau) d x+\int_{0}^{\tau} \int_{-\infty}^{\infty} w_{t}^{2} d x d t=O(1) \int_{-\infty}^{\infty} w_{t}^{2}(x, 0) d x \\
& +O(1) \int_{0}^{\tau} \int_{-\infty}^{\infty}\left(w_{t x}^{2}+\sum_{j=1}^{2}\left|\nabla^{j} z\right|^{2}+\varepsilon w_{x}^{2}+\varepsilon \lambda_{* x}\left(z^{2}+w^{2}\right)+\left|\lambda_{* x}\right|^{3}\right) d x d t
\end{aligned}
$$

$$
\begin{aligned}
& \int_{\infty}^{\infty} w_{t t}^{2}(x, \tau) d x+\int_{0}^{\tau} \int_{-\infty}^{\infty} w_{t t}^{2} d x d t=O(1) \int_{-\infty}^{\infty} w_{t t}^{2}(x, 0) d x \\
& +O(1) \int_{0}^{\tau} \int_{-\infty}^{\infty}\left(w_{x t t}^{2}+\sum_{j=1}^{3}\left|\nabla^{j} z\right|^{2}+\varepsilon\left(\sum_{j=1}^{2}\left|\nabla^{j} w\right|^{2}+\lambda_{* x}\left(z^{2}+w^{2}\right)\right)+\left|\lambda_{* x}\right|^{3}\right) d x d t
\end{aligned}
$$

From $(3.5)_{2 t t}$ we have

and so

$$
w_{t t t}=-[g]_{x t t}+[h]_{t t}-F_{t t},
$$

$$
\begin{aligned}
& \int_{\infty}^{\infty} w_{t t t}^{2}(x, \tau) d x+\int_{0}^{\tau} \int_{-\infty}^{\infty} w_{t t t}^{2} d x d t \\
& =O(1) \int_{-\infty}^{\infty}\left(w_{x t t}^{2}+\sum_{j=1}^{3}\left|\nabla^{j} z\right|^{2}+w_{t t}^{2}+\varepsilon\left(\sum_{j=0}^{1}\left|\nabla^{j} w_{x}\right|^{2}+\lambda_{* x}\left(z^{2}+w^{2}\right)\right)+\left|\lambda_{* x}\right|^{3}\right)(x, \tau) d x \\
& \quad+O(1) \int_{0}^{\tau} \int_{-\infty}^{\infty}\left(w_{x t t}^{2}+\sum_{j=1}^{3}\left|\nabla^{j} w\right|^{2}+w_{t t}^{2}\right. \\
& \left.\quad+\varepsilon\left(\sum_{j=0}^{1}\left|\nabla^{j} w_{x}\right|^{2}+\lambda_{* x}\left(z^{2}+w^{2}\right)\right)+\left|\lambda_{* x}\right|^{3}\right) d x d t
\end{aligned}
$$


By integrating the differentials of (3.7) we have

$$
\begin{aligned}
& \int_{-\infty}^{\infty} \sum_{j=0}^{2}\left(\left|\nabla^{j} w_{x}\right|^{2}(x, \tau) d x\right) \\
& =O(1) \int_{-\infty}^{\infty}\left(\sum_{j=0}^{2}\left(\left|\nabla^{j} z\right|^{2}+\varepsilon\left(\sum_{j=0}^{2}\left(\left|\nabla^{j} w\right|^{2}+\left|\lambda_{* x}\right|^{3}\right) d x\right)\right)\right)(x, \tau) d x, \\
& \int_{0}^{\tau} \int_{-\infty}^{\infty} \sum_{j=0}^{2}\left(\left|\nabla^{j} w_{x}\right|^{2} d x d t\right) \\
& =O(1) \int_{0}^{\tau} \int_{-\infty}^{\infty}\left(\sum_{j=1}^{3}\left(\left|\nabla^{j} z\right|^{3}+\varepsilon\left(\lambda_{* x}\left(z^{2}+w^{2}\right)\right)\right)+\sum_{j=0}^{2}\left(\left|\nabla^{j} w\right|^{2}\right)+\left|\lambda_{* x}\right|^{3}\right) d x d t
\end{aligned}
$$

From (3.17) it follows that

$$
\begin{aligned}
& \int_{-\infty}^{\infty} w^{2}(x, \tau) d x=O(1) \int_{-\infty}^{\infty}\left(|\nabla w|^{2}+z^{2}+\left|z_{x}\right|^{2}+\mu_{x}^{4}\right)(x, \tau) d x, \\
& \int_{0}^{\tau} \int_{-\infty}^{\infty} \lambda_{* x} w^{2} d x d t=O(1) \int_{0}^{\tau} \int_{-\infty}^{\infty}\left(\lambda_{* x} z^{2}+|\nabla(z, w)|^{2}+\left|\lambda_{* x}\right|^{3}\right) d x d t .
\end{aligned}
$$

We have from $C^{-1}\left((3.19)_{1}+(3.19)_{2}+(3.19)_{3}\right)+(3.19)_{4}+\ldots+(3.19)_{7}, C$ a large number, that for $\varepsilon \ll 1$, (3.13),

$$
\begin{aligned}
\int_{-\infty}^{\infty} \sum_{j=0}^{3}\left|\nabla^{j} w_{x}\right|^{2}(x, \tau) d x+\int_{0}^{\tau} \int_{-\infty}^{\infty}\left(\sum_{j=1}^{3}\left(\left|\nabla^{j} w\right|^{2}+\lambda_{* x} w^{2}\right) d x d t\right) \\
=O(1) \int_{-\infty}^{\infty}\left(\sum_{j=0}^{3}\left(\left|\nabla^{j} z\right|^{2}(x, \tau)\right)\right) \\
\quad+\sum_{j=0}^{3}\left|\nabla^{j}(x, w)\right|^{2}(x, 0) d x+\int_{0}^{\tau} \int_{-\infty}^{\infty}\left(\sum_{j=1}^{3}\left(\left|\nabla^{j} z\right|^{2}+\lambda_{* x} z^{2}+\left|\lambda_{* x}\right|^{3}\right) d x d t\right) .
\end{aligned}
$$

Finally, we have from $(3.20)+C^{-5}(3.18)_{1}+C^{-4}(3.18)_{2}+C^{-3}(3.18)_{3}$ $+C^{-3}(3.18)_{4}+C^{-2}(3.18)_{5}+C^{-1}(3.18)_{6}+(3.18)_{7}+(3.18)_{8}, C$ a large constant, that for $\varepsilon \ll 1,(3.13)$,

$$
\begin{aligned}
& \int_{-\infty}^{\infty} \sum_{j=0}^{3}\left(\left|\nabla^{j}(z, w)\right|^{2}(x, \tau) d s+\int_{0}^{\tau} \int_{-\infty}^{\infty}\left(\lambda_{* x}\left(z^{2}+w^{2}\right)+\sum_{j=1}^{3}\left(\left|\nabla^{j}(z, w)\right|^{2}\right) d x d t\right)\right) \\
& =O(1) \int_{-\infty}^{\infty} \sum_{j=1}^{3}\left(\left|\nabla^{j}(z, w)\right|^{2}\right)(x, 0) d x+O(1) \int_{0}^{\tau} \int_{-\infty}^{\infty}\left|\lambda_{* x}\right|^{3} d x d t
\end{aligned}
$$

With (3.21), the hypothesis (3.13) clearly holds. The global existence of the solution follows from the standard local existence theory and the energy estimate (3.21). It also follows that $\int_{-\infty}^{\infty}\left(z_{w}^{2}+w_{x}^{2}\right)(x, t) \rightarrow 0$ as $t \rightarrow \infty$, whence we have,

$$
\begin{aligned}
z^{2}(x, t)+w^{2}(x, t) & =\int_{-\infty}^{\infty}\left(2 z z_{x}+2 w w_{x}\right)(y, t) d y \\
& \leqq\left(\int_{-\infty}^{\infty}\left(z^{2}+w^{2}\right)(x, t) d x\right)\left(\int_{-\infty}^{\infty}\left(z_{x}^{2}+w_{x}^{2}\right)(x, t) d x\right) \rightarrow 0 \text { as } t \rightarrow \infty
\end{aligned}
$$

This completes the proof of the theorem. Q.E.D. 


\section{Admissible Traveling Waves}

Suppose that $(u, v)(x, t)=(\varphi, \psi)(x-\sigma t)$ is a traveling wave for (1.1). A traveling wave is a permanent wave form connecting constant states $\left(u_{ \pm}, v_{ \pm}\right)$ $\equiv(\varphi, \psi)( \pm \infty)$. Since $\left(u_{ \pm}, v_{ \pm}\right)$are constant, and the only constant states solution of (1.1) are equilibrium states, we have

$$
v_{ \pm}=v_{*}\left(u_{ \pm}\right) \text {. }
$$

From the conservation law in (1.1) we have

$$
\sigma\left(u_{+}-u_{-}\right)=f\left(u_{+}, v_{+}\right)-f\left(u_{-}, v_{-}\right) .
$$

From (4.1) and (4.2) we see that, cf. (1.3),

$$
\sigma\left(u_{+}-u_{-}\right)=f_{*}\left(u_{+}\right)-f_{*}\left(u_{-}\right) .
$$

That is, $\left(u_{-}, u_{+}\right)$also satisfies the jump condition for the equilibrium equation (1.3). The following theorem relates the admissibility of shock waves for the scalar conservation law (1.3) to the existence and stability of traveling waves for the relaxation model (1.1).

Theorem 4.1. Suppose that $(\varphi, \psi)(x-\sigma t)$ is a smooth traveling wave of (1.1) and that it is stable in the sense that the speed is subcharacteristic,

$$
\lambda_{1}((\varphi, \psi)(\xi))<\sigma<\lambda_{2}((\varphi, \psi)(\xi))
$$

for $\xi \in \mathbb{R}^{1}$. Then the corresponding shock wave $\left(u_{-}, u_{+}\right), u_{ \pm} \equiv \varphi( \pm \infty)$, for (1.3) is admissible in the sense that

$$
\frac{f_{*}\left(u_{+}\right)-f_{*}\left(u_{-}\right)}{u_{+}-u_{-}}<\frac{f_{*}(u)-f_{*}\left(u_{-}\right)}{u-u_{-}}
$$

for all $u$ strictly between $u_{-}$and $u_{+}$. Conversely, given an admissible shock wave $\left(u_{-}, u_{+}\right)$of (1.3) such that its speed $\sigma$ differs from $\lambda_{i}(u, v), i=1,2$, along $\sigma u-f(u, v)$ $=\sigma u_{ \pm}-f\left(u_{ \pm}, v_{ \pm}\right), v_{ \pm} \equiv v_{*}\left(u_{ \pm}\right)$, between $u_{-}$and $u_{+}$, then there exists a stable smooth traveling wave $(\varphi, \psi)(x-\sigma t)$ of $(1.1)$, and the end states $(\varphi, \psi)( \pm \infty)$ $=\left(u_{ \pm}, v_{ \pm}\right)$satisfy (4.4).

Proof. From (1.1) a traveling wave $(\varphi, \psi)(x-\sigma t)$ satisfies

$$
\begin{gathered}
-\sigma \varphi^{\prime}+f(\varphi, \psi)^{\prime}=0, \\
-\sigma \psi^{\prime}+g(\varphi, \psi)^{\prime}=h(\varphi, \psi) .
\end{gathered}
$$

Integrate (4.6) to obtain

$-\sigma \varphi+f(\varphi, \psi)=a, \quad a \equiv-\sigma u_{ \pm}+f\left(u_{ \pm}, v_{ \pm}\right), \quad\left(u_{ \pm}, v_{ \pm}\right) \equiv(\varphi, \psi)( \pm \infty)$.

The equilibrium set

$$
\Gamma \equiv\left\{(u, v): v=v_{*}(u)\right\}
$$


is a curve, (1.2). From (4.1), $\left(u_{ \pm}, v_{ \pm}\right) \in \Gamma$. Along the traveling wave $(\varphi, \psi)$ we have from (1.4), (1.7), (4.6) and (4.7) that

$$
\begin{gathered}
\varphi^{\prime}=\frac{-f_{v} h}{\left(\lambda_{1}-\sigma\right)\left(\lambda_{2}-\sigma\right)}, \\
\psi^{\prime}=\frac{\left(f_{u}-\sigma\right) h}{\left(\lambda_{1}-\sigma\right)\left(\lambda_{2}-\sigma\right)}, \\
h^{\prime}=\frac{(\mu-\sigma) h_{v} h}{\left(\lambda_{1}-\sigma\right)\left(\lambda_{2}-\sigma\right)}, \\
\left(\varphi-v_{*}(\varphi)\right)^{\prime}=\frac{\left(f_{u}+v_{*}^{\prime} f_{v}-\sigma\right) h}{\left(\lambda_{1}-\sigma\right)\left(\lambda_{2}-\sigma\right)} .
\end{gathered}
$$

Suppose $(\varphi, \psi)$ is a smooth traveling wave satisfying (4.4). Then from (2.8), (4.9), and (4.10) we see that the only critical points for the autonomous o.d.e. (4.6) and (4.7) are those on $\Gamma$, where $h=0$. Consequently $(\varphi, \psi)(\xi) \notin \Gamma$ for $-\infty<\xi<\infty$, and $\varphi(\xi)$, $-\infty<\xi<\infty$, is monotone and lies between the limiting values $u_{-}$and $u_{+}$. Suppose that for some $u$ between $u_{-}$and $u_{+}$,

$$
\sigma=\frac{f_{*}\left(u_{+}\right)-f_{*}\left(u_{-}\right)}{u_{+}-u_{-}}=\frac{f_{*}(u)-f_{*}\left(u_{-}\right)}{u-u_{-}} .
$$

Then by (4.8) we have for some $\xi \in(-\infty, \infty)$ with $\varphi(\xi)=u$,

$$
f\left(u, v_{*}(u)\right) \equiv f_{*}(u)=f(\varphi(\xi), \psi(\xi))=f(u, \psi(\xi)) .
$$

And so from (2.8), $v_{*}(u)=\psi(\xi)$. In other words $(\varphi, \psi)(\xi) \in \Gamma$, which is a contradiction. Thus to prove (4.5) it remains to show that the inequality holds for $\xi \cong-\infty$. Since at $\xi=-\infty,(\varphi, \psi)=\left(u_{-}, v_{-}\right) \in \Gamma$, we have from (1.2) that $\left(\varphi-v_{*}(\varphi)\right)$ and $h$ have opposite signs. Consequently we have from (4.4) and (4.12) that $\sigma<\lambda_{*}$ near $\xi=-\infty$. This proves (4.5) near $\xi=-\infty$ because

$$
\lim _{u \rightarrow u_{-}} \frac{f_{*}(u)-f_{*}\left(u_{-}\right)}{u-u_{-}}=\lambda_{*}\left(u_{-}\right) .
$$

This completes the proof of the first part of Theorem 4.1; the second part is proved by similar arguments. Q.E.D.

The above theorem completely characterizes traveling waves of (1.1) whose speed is subcharacteristic, (4.4). From the study of the stability of equilibrium states in the last two sections, we see that it is necessary to assume the equilibrium characteristic $\lambda_{*}$ to be subcharacteristic on the equilibrium set $\Gamma$

$$
\lambda_{1}\left(u, v_{*}(u)\right)<\lambda_{*}(u)<\lambda_{2}\left(u, v_{*}(u)\right) .
$$

With (4.13) it follows that for weak traveling waves of (1.1), the speed $\sigma$ is close to $\lambda_{*}$ and thereby is subcharacteristic. However, when a traveling wave is strong, its speed may fail to be subcharacteristic. In this case, a traveling wave will have to contain discontinuity waves. This issue will be taken up when we study the admissible time-asymptotic wave pattern in the last section. In the next section we show that weak traveling waves are nonlinearly stable. 


\section{Stability of Traveling Waves}

Let $(\varphi, \psi)(x-\sigma t)$ be a smooth traveling wave of $(1.1)$ with speed $\sigma$, and $(u, v)(x, t)$ be a solution of (1.1) whose initial data is a perturbation of $(\varphi, \psi)$ :

$$
(u, v)(x, 0)=(\varphi, \psi)(x)+(\bar{u}, \bar{v})(x, 0), \quad(\bar{u}, \bar{v})( \pm \infty, 0)=0 .
$$

Our purpose is to show that $(u, v)(x, t)$ tends to $(\varphi, \psi)\left(x+x_{0}-\sigma t\right)$ as $t \rightarrow \infty$. The amount of translation $x_{0}$ due to the perturbation $(\bar{u}, \bar{v})$ can be identified from the conservation law in (1.1):

$$
\int_{-\infty}^{\infty}(u(x, t)-\varphi(x-\sigma t)) d x=\int_{-\infty}^{\infty} \bar{u}(x, 0) d x .
$$

Since $u(x, t) \rightarrow \varphi\left(x+x_{0}-\sigma t\right)$, we have

$$
\begin{aligned}
0 & =\int_{-\infty}^{\infty} u(x, t)-\varphi\left(x+x_{0}-\sigma t\right) d x \\
& =\int_{-\infty}^{\infty} \bar{u}(x, 0) d x-\int_{-\infty}^{\infty}\left(\varphi\left(x+x_{0}-\sigma t\right)-\varphi(x-\sigma t)\right) d x \\
& =\int_{-\infty}^{\infty} \bar{u}(x, 0) d x-x_{0}\left(u_{+}-u_{-}\right)
\end{aligned}
$$

and

$$
x_{0}=\left(u_{+}-u_{-}\right)^{-1} \int_{-\infty}^{\infty} \bar{u}(x, 0) d x, \quad u_{ \pm} \equiv \varphi( \pm \infty) .
$$

From here on, we assume that $x_{0}=0$ through the translation of $(\varphi, \psi)(x-\sigma t)$ to $(\varphi, \psi)\left(x+x_{0}-\sigma t\right)$. Thus we have

$$
\int_{-\infty}^{\infty}(u(x, t)-\varphi(x-\sigma t)) d x=0 .
$$

With (5.3) it is natural and convenient to write

$$
\begin{gathered}
u(x, t)=\varphi(x-\sigma t)+z_{x}(x, t), \quad v(x, t)=\psi(x-\sigma t)+w(x, t), \\
z(x, t) \equiv \int_{-\infty}^{x} u(y, t)-\varphi(y-\sigma t) d y, \quad z( \pm \infty, t)=0 .
\end{gathered}
$$

The last identity comes from (5.3). Since both $(u, v)$ and $(\varphi, \psi)$ satisfy (1.1), we have, by integrating with respect to $x$ the difference of conservation law in (1.1) for $u$ and $\psi$, and by simply taking the difference of the rate equation in (1.2) for $v$ and $\psi$,

$$
\begin{gathered}
z_{t}+f\left(z_{x}+\varphi, w+\psi\right)-f(\varphi, \psi)=0, \\
w_{t}+\left(g\left(z_{x}+\varphi, w+\psi\right)-g(\varphi, \psi)\right)_{x}=h\left(z_{x}+\varphi, w+\psi\right)-h(\varphi, \psi) .
\end{gathered}
$$

From $(5.5)_{1}$ and (2.8) we may represent $w$ in terms of $z$ :

$$
\begin{gathered}
w=f_{v}^{-1}\left(-z_{t}-f_{u} z_{x}-Q_{0}(f)\right), \\
Q_{0}(f) \equiv f\left(z_{x}+\varphi, w+\psi\right)-f_{u} z_{x}+f_{v} w=O(1)\left(z_{x}^{2}+w^{2}\right), \\
f_{u} \equiv f_{u}(\varphi, \psi) \quad \text { etc. }
\end{gathered}
$$


and for $z_{x}^{2}+w^{2}$ small,

$$
w=W\left(z_{t}, z_{x}, \varphi, \psi\right) O(1)\left(\left|z_{t}\right|+\left|z_{x}\right|\right)
$$

for some smooth function $W$. Plug (5.6) into $-f_{v}(5.5)_{2}$, we have

$$
\begin{aligned}
z_{t t} & \left.+\left(\lambda_{1}+\lambda_{2}\right) z_{x t}+\lambda_{1} \lambda_{2} z_{x x}-h_{v}\left(z_{t}+\mu z_{x}\right)=f_{v}^{-1} f_{v t}+f_{v}^{-1} g_{v} f_{v x}-g_{v x}\right)\left(z_{t}+f_{u} z_{x}\right) \\
& +Q_{0}(f)\left(f_{v}^{-1} g_{v} f_{v x}-g_{v x}+h_{v}\right)-f_{v} Q_{0}(h)-Q_{0}(f)_{t}-g_{v} Q_{0}(f)_{x}+f_{v} Q_{0}(g)_{x},
\end{aligned}
$$

where the functions $\lambda_{1}, \lambda_{2}, f_{u}$, etc. are evaluated at $(\varphi, \psi)$ and $Q_{0}$ is a higher-order term defined in (5.6). The right-hand side of (5.8) are higher-order terms. The left hand side contains a first-order term with speed

$$
\mu \equiv f_{u}-f_{v} h_{u} h_{v}^{-1},
$$

which is the dynamic subcharacteristic speed and plays the basic role of governing the large-time behavior of the weak waves traveling over the traveling wave $(\varphi, \psi)$. For an equilibrium state $\left(u, v_{*}(u)\right)$, we have from differentiating (1.2) that

$$
h_{u}+h_{v} v_{*}=0
$$

and so from (1.3) and (1.5),

$$
\mu=f_{u}-f_{v} h_{u} h_{v}^{-1}=f_{u}+f_{v} v_{*}=\lambda_{*} .
$$

In other words, the dynamics speed $\mu$ equals the equilibrium speed $\lambda_{*}$ when the state is in equilibrium. Since a traveling wave $(\varphi, \psi)$ is not in equilibrium except at end states $x= \pm \infty, \mu$ differs from $\lambda_{*}$ in general.

The stability of a traveling wave is the consequence of three things. One is that the speed is subcharacteristic.

$$
\lambda_{1}<\sigma<\lambda_{2}
$$

As we have seen in Theorem 4.1, this is equivalent to the admissibility of the corresponding shock wave for the equilibrium equation (1.3). The second is the nonlinearity of the system (1.1). The simplest nonlinearity is that characteristic speed is strictly monotone across the wave. For simplicity, we will assume that $f_{*}(u)$ is convex so that $(4.5)$ is equivalent to $\lambda_{*}(\varphi, \psi)_{x}<0$. However, the proper characteristic here is $\mu$ and not $\lambda_{*}$. For weak traveling waves $\mu$ is close to $\lambda_{*}$, and we have

$$
\left|\varphi_{x}\right|+\left|\psi_{x}\right| \leqq C \mu_{x} \ll 1 ; \quad|\sigma-\mu|+\left|\sigma-\lambda_{*}\right| \ll 1
$$

for some positive constant $C$. The third element for the stability is that (1.1) is a relaxation model, (1.2). Our stability analysis can be refined to handle any weak traveling waves when $f_{*}$ may have isolated inflection points. We will not elaborate this here though.

Theorem 5.1. Suppose that $(\varphi, \psi)(x-\sigma t)$ is a smooth traveling wave of (1.1) satisfying (5.10) and (5.11). Then any perturbation (5.1) of $(\varphi, \psi)$ gives rise to global solution $(u, v)$ of $(1.1)$ which tends to $(\varphi, \psi)\left(x+x_{0}-\sigma t\right)$ uniformly in $x$ as $t \rightarrow \infty$, provided that $\varepsilon(0)$ is sufficiently small, where

$$
\varepsilon(\tau) \equiv\left|u_{+}-u_{-}\right|+\max _{0 \leqq t \leqq \tau} \int_{-\infty}^{\infty}\left(\sum_{j=0}^{3}\left|\frac{\partial^{j} z}{\partial x^{j}}(x, t)\right|^{2}+\sum_{j=0}^{2}+\left|\frac{\partial^{j} w}{\partial x^{j}}(x, t)\right|^{2}\right) d x .
$$


Proof. Since the functions $\lambda_{1}, \lambda_{2}, f_{u}$, etc. in (5.8) are functions of $(\varphi, \psi)(x-\sigma t)$, it is convenient to change the variables $(x, t)$ to $(\xi, t), \zeta=x-\sigma t$, and (5.8) becomes

$$
\begin{aligned}
z_{t t}+ & \left(\lambda_{1}+\lambda_{2}-2 \sigma\right) z_{\xi t}+\left(\sigma-\lambda_{1}\right)\left(\sigma-\lambda_{2}\right) z_{\xi \xi}-h_{v}\left(z_{t}-\sigma z_{\xi}+\mu z_{\xi}\right) \\
= & \left(\sigma f_{v}^{-1} f_{v \xi}+f_{v}^{-1} g_{v} f_{v \xi}-g_{v \xi}-g_{v \xi}\right)\left(z_{t}-\sigma z_{\xi}+f_{u} z_{\xi}\right) \\
& +Q_{0}(f)\left(f_{v}^{-1} g_{v} f_{v \xi}-g_{v \xi}+h_{v}\right)-f_{v} Q_{0}(h)-Q_{0}(f)_{t}+\sigma Q_{0}(f)_{\xi} \\
& -g_{v} Q_{0}(f)_{\xi}+f_{v} Q_{0}(g)_{\xi} \equiv \text { R.H.S. }
\end{aligned}
$$

All known functions $\lambda_{1}, \lambda_{2}, f_{u}$, etc. are functions of $\xi$ only. We now perform the energy estimate much the same way as in the proof of Theorem 3.1. There are two notable differences, however. A rarefaction wave is expanding, (3.12), and rarefying (3.2), while a traveling wave is compressive (5.11) (or, in the general case, mostly compressive) and maintains a definite shape. These differences are matched by a major difference between Eqs. (3.9) and (5.12) in that on the left-hand side of (3.9) the equilibrium subcharacteristic speed $\lambda_{*}$ is differentiated, while in (5.12) the dynamic subcharacteristic speed $\mu$ is not. This is because we use the conservation law (5.3) in deriving (5.12); while the convergence to a rarefaction wave is in general not in $L_{1}$, and the conservation law is of little use in deriving (3.9). Integrate (5.12)z over $0 \leqq t \leqq \tau,-\infty<x \leqq \infty$ and use (1.2), (5.10), (5.11) to obtain

$$
\begin{aligned}
& \frac{1}{2} \int_{-\infty}^{\infty}\left(\left|h_{v}\right| z^{2}\right)(x, \tau) d x+\int_{0}^{\tau} \int_{-\infty}^{\infty}\left(\frac{1}{2}\left|h_{v}\right|\left|\mu_{\xi}\right| z^{2}+\left|\left(\sigma-\lambda_{1}\right)\left(\sigma-\lambda_{2}\right)\right| z_{\xi}^{2}\right) d x d t \\
& =\frac{1}{2} \int_{-\infty}^{\infty}\left(\left(\left|h_{v}\right| z^{2}\right)(x, 0)+\left(z_{t} z\right)(x, 0)-\left(z_{t} z\right)(x, \tau)\right) d x \\
& \quad+\int_{0}^{\tau} \int_{-\infty}^{\infty}\left(z_{t}^{2}+\left(\lambda_{1}+\lambda_{2}-2 \sigma\right)_{\xi} z_{t} z+\left(\lambda_{1}+\lambda_{2}-2 \sigma\right) z_{t} z_{\xi}\right. \\
& \left.\quad+\left(\left(\sigma-\lambda_{1}\right)\left(\sigma-\lambda_{2}\right)\right)_{\xi} z_{\xi} z+\text { R.H.S. }\right) d x d t .
\end{aligned}
$$

Under the assumption that

$$
\max _{0 \leqq t \leqq \tau} \varepsilon(t) \ll 1,
$$

the above identify and (5.11) yield

$$
\begin{aligned}
& \int_{-\infty}^{\infty} z^{2}(x, \tau) d x+\int_{0}^{\tau} \int_{-\infty}^{\infty}\left(\left|\mu_{\xi}\right| z^{2}+z_{\xi}^{2}\right) d x d t \\
& \quad=O(1) \int_{-\infty}^{\infty}\left(z^{2}(x, 0)+z_{t}^{2}(x, 0)+z_{t}^{2}(x, \tau)\right) d x+O(1) \int_{0}^{\tau} \int_{-\infty}^{\infty}\left(z_{t}^{2}+\text { R.H.S. }\right) d x d t .
\end{aligned}
$$

From (5.6) and (5.7) we have

$$
\mid Q_{0}\left(f|+| Q_{0}(g)|+| Q_{0}(h) \mid=O(1)\left(\left|z_{t}\right|^{2}+\left|z_{\xi}\right|^{2}\right) .\right.
$$

By the same arguments in deriving (5.14) we have from (5.14) and (5.15) that

$$
\begin{aligned}
& \int_{-\infty}^{\infty} z^{2}(x, \tau) d x+\int_{0}^{\tau} \int_{-\infty}^{\infty}\left(\left|\mu_{\xi}\right| z^{2}+z_{\xi}^{2}\right) d x d t \\
& \quad=O(1) \int_{-\infty}^{\infty}\left(z^{2}(x, 0)+z_{t}^{2}(x, 0)+z_{t}^{2}(x, \tau) d x+O(1) \int_{0}^{\tau} \int_{-\infty}^{\infty} z_{t}^{2} d x d t .\right.
\end{aligned}
$$

Without going into details, we conclude from integrating $(5.12) z_{t},(5.12)_{\xi} z_{\xi}$, $(5.12)_{t} z_{t t},(5.12)_{\xi} z_{t \xi},(5.12)_{\xi \xi} z_{\xi \xi},(5.12)_{\xi \xi} z_{\xi \xi t},(5.12)_{t \xi} z_{t t \xi}$, and (5.12) $)_{t t} z_{t t}$, respectively, 
that

$$
\begin{aligned}
& \int_{-\infty}^{\infty} z_{t}^{2}(x, \tau) d x+\int_{0}^{\tau} \int_{-\infty}^{\infty} z_{t}^{2} d x d t \\
& =O(1) \int_{-\infty}^{\infty} z_{t}^{2}(x, 0) d x+O(1) \int_{0}^{\tau} \int_{-\infty}^{\infty}\left(z_{\xi \xi}^{2}+\varepsilon\left(z_{\xi}^{2}+z_{\xi t}^{2}+z_{t t}^{2}\right)\right) d x d t, \\
& \int_{-\infty}^{\infty} z_{\xi}^{2}(x, \tau) d x+\int_{0}^{\tau} \int_{-\infty}^{\infty} z_{\xi \xi}^{2} d x d t \\
& =O(1) \int_{-\infty}^{\infty} z_{\xi}^{2}(x, 0) d x+O(1) \int_{0}^{\tau} \int_{-\infty}^{\infty}\left(z_{t t}^{2}+z_{t \xi}^{2}+\varepsilon|\nabla z|^{2}\right) d x d t, \\
& \int_{-\infty}^{\infty} z_{t t}^{2}(x, \tau) d x+\int_{0}^{\tau} \int_{-\infty}^{\infty} z_{t t}^{2} d x d t \\
& =O(1) \int_{-\infty}^{\infty} z_{t t}^{2}(x, 0) d x+O(1) \int_{0}^{\tau} \int_{-\infty}^{\infty}\left(z_{t t \xi}^{2}+z_{t \xi \xi}^{2}+\varepsilon\left(|\nabla z|^{2}+z_{t \xi}^{2}+z_{\xi \xi}^{2}\right)\right) d x d t, \\
& \int_{-\infty}^{\infty}\left(z_{t \xi}^{2}+z_{\xi \xi}^{2}\right)(x, \tau) d x+\int_{0}^{\tau} \int_{-\infty}^{\infty} z_{t \xi}^{2} d x d t \\
& =O(1) \int_{-\infty}^{\infty}\left(z_{t \xi}^{2}+z_{\xi \xi}^{2}(x, 0) d x+O(1) \int_{0}^{\tau} \int_{-\infty}^{\infty}\left(z_{t \xi \xi}^{2}+\varepsilon\left(|\nabla z|^{2}+z_{t t}^{2}+z_{\xi \xi}^{2}\right) d x d t,\right.\right. \\
& \int_{-\infty}^{\infty}\left(z_{\xi \xi}^{2}(x, \tau) d x+\int_{0}^{\tau} \int_{-\infty}^{\infty} z_{\xi \xi \xi}^{2} d x d t\right. \\
& =O(1) \int_{-\infty}^{\infty}\left(z_{\xi \xi}^{2}(x, 0) d x\right)+O(1) \int_{0}^{\tau} \int_{-\infty}^{\infty}\left(z_{t \xi \xi}^{2}+\varepsilon\left(|\nabla z|^{2}+\left|\nabla^{2} z\right|^{2}+z_{t t \xi}^{2}\right)\right) d x d t, \\
& \int_{-\infty}^{\infty}\left(z_{t \xi \xi}^{2}+z_{\xi \xi \xi}^{2}\right)(x, \tau) d x+\int_{0}^{\tau} \int_{-\infty}^{\infty} z_{\xi \xi t}^{2} d x d t \\
& =O(1) \int_{-\infty}^{\infty}\left(z_{t \xi \xi}^{2}+z_{\xi \xi \xi}^{2}(x, 0) d x+O(1) \int_{0}^{\tau} \int_{-\infty}^{\infty} \varepsilon\left(|\nabla z|^{2}+\left|\nabla^{2} z\right|^{2}+z_{\xi \xi \xi}^{2}\right) d x d t\right. \\
& +O(1) \int_{0}^{\tau} \int_{-\infty}^{\infty}\left(-Q_{0}(f)_{t}+\sigma Q_{0}(f)_{\xi}-g_{v} Q_{0}(f)_{\xi}+f_{v} Q_{0}(g)_{\xi}\right)_{\xi \xi} z_{\xi \xi t} d x d t \\
& \int_{-\infty}^{\infty} z_{t t \xi}^{2}(x, \tau) d x+\int_{0}^{\tau} \int_{-\infty}^{\infty} z_{t \xi \xi}^{2} d x d t \\
& =O(1) \int_{-\infty}^{\infty}\left(z_{t t \xi}^{2}(x, 0) d x+O(1) \int_{0}^{\tau} \int_{-\infty}^{\infty} \varepsilon|\nabla z|^{2}+\left|\nabla^{2} z\right|^{2}+z_{\xi \xi \xi}^{2}\right) d x d t \\
& +O(1) \int_{0}^{\tau} \int_{-\infty}^{\infty}\left(-Q_{0}(f)_{t}+\sigma Q_{0}(f)_{\xi}-g_{v} Q_{0}(f)_{\xi}+f_{v} Q_{0}(g)_{\xi}\right)_{t \xi} z_{t t \xi} d \xi d t,
\end{aligned}
$$

and

$$
\begin{aligned}
& \int_{-\infty}^{\infty} z_{t t t}^{2}(x, \tau) d x+\int_{0}^{\tau} \int_{-\infty}^{\infty} z_{t t t}^{2} d x d t \\
& =O(1) \int_{-\infty}^{\infty} z_{t t t}^{2}(x, 0) d x+O(1) \int_{0}^{\tau} \int_{-\infty}^{\infty} \varepsilon\left(|\nabla z|^{2}+\left|\nabla^{2} z\right|^{2}+z_{t t \xi}^{2}\right) d x d t \\
& \quad+O(1) \int_{0}^{\tau} \int_{-\infty}^{\infty}\left(-Q_{0}(f)_{t}+\sigma Q_{0}(f)_{\xi}-g_{v} Q_{0}(f)_{\xi}+f_{v} Q_{0}(g)_{\xi}\right)_{t t} z_{t t t} d x d t
\end{aligned}
$$


The above estimates treat all the first three derivatives of $z$. Note, however, the last integrals in (5.16) $)_{7}-(5.16)_{9}$ appear to contain fouth derivatives of $z$, cf. (5.15). This difficulty is resolved by Taylor expansions and integration by parts. We illustrate this by considering the first such integral using (5.6) and (5.7),

$$
\begin{aligned}
\int_{0}^{\tau} \int_{-\infty}^{\infty}-Q_{0}(f)_{t t \xi} z_{t t \xi} d \xi d t= & \left.-\int_{0}^{\tau} \int_{-\infty}^{\infty}\left(\left[f_{u}\right] z_{t t \xi \xi}+\left[f_{v}\right] w_{t t \xi}\right) z_{t t \xi}+\text { L.O.T. }\right) d x d t \\
= & -\int_{0}^{\tau} \int_{-\infty}^{\infty}\left(\left[f_{u}\right] z_{t t \xi \xi}+\left[f_{v}\right]\left(W_{111} z_{t t t \xi}\right.\right. \\
& \left.\left.\left.+W_{222} z_{t t \xi \xi}\right)\right) z_{t t \xi}+\text { L.O.T. }\right) d x d t
\end{aligned}
$$

where $W_{111}\left(W_{222}\right)$ is the third derivative of the function $W$ in (5.7) with respect to the first (second) variable and L.O.T. are lower-order terms and not including fourth derivatives of $z$. The term L.O.T. are absorbed into the left-hand sides of (5.16). We have by parts

$$
\begin{aligned}
- & \int_{0}^{\tau} \int_{-\infty}^{\infty}\left(\left[f_{u}\right] z_{t t \xi \xi}+\left[f_{v}\right]\left(W_{111} z_{t t t \xi}+W_{222} z_{t t \xi \xi}\right) z_{t t \xi} d x d t\right. \\
= & \int_{0}^{\tau} \int_{-\infty}^{\infty}\left(\frac{1}{2}\left[f_{u}\right]_{\xi} z_{t t \xi}^{2}+\frac{1}{2}\left(\left[f_{v}\right] W_{111}\right)_{t} z_{t t \xi}^{2}+\frac{1}{2}\left(W_{222}\right)_{\xi} z_{t t \xi}^{2}\right) d x d t \\
& +\int_{-\infty}^{\infty}\left(\frac{1}{2}\left[f_{v}\right] W_{11} z_{t t \xi}^{2}(x, \tau)-\frac{1}{2}\left[f_{v}\right] W_{111} z_{t t \xi}^{2}(x, 0)\right) d x .
\end{aligned}
$$

Since $\left[f_{u}\right],\left[f_{v}\right], W_{111}$ and $W_{222}$ are functions of $z_{t}, z_{x}, \varphi$ and $\psi$, the above expression involves only up to the third derivatives of $z$. Similar treatment applies to other terms, and we conclude that the last integrals in $(5.16)_{7}-(5.16)_{9}$ are

$$
\begin{aligned}
& O(1) \int_{0}^{\tau} \int_{-\infty}^{\infty} \varepsilon\left(|\nabla z|^{2}+\left|\nabla^{2} z\right|^{2}+\left|\nabla^{3} z\right|^{2}\right) d x d t \\
& \quad+O(1) \varepsilon \int_{-\infty}^{\infty}\left(\sum_{j=1}^{3}\left|\nabla^{j} z\right|^{2}(x, 0)+\sum_{j=0}^{3}\left|\nabla^{j} z\right|^{2}(x, \tau)\right) d x .
\end{aligned}
$$

With this, we conclude from $C^{-4}(5.16)_{1}+C^{-3}(5.16)_{2}+C^{-2}(5.16)_{3}+C^{-1}(5.16)_{4}$ $+C^{-1}(5.16)_{6}+C^{-1}(5.16)_{6}+(5.16)_{7}+(5.16)_{8}+(5.16)_{9}, C$ is a sufficiently large constant, that under (5.13) we have

$$
\begin{array}{r}
\int_{-\infty}^{\infty} \sum_{j=0}^{3}\left|\nabla^{j} z(x, \tau)\right|^{2} d x+\int_{0}^{\tau} \int_{-\infty}^{\infty}\left(\left|\mu_{x}\right| z^{2}+\sum_{j=1}^{3}\left|\nabla^{j} z\right|^{2}\right) d x d t \\
=O(1) \int_{-\infty}^{\infty} \sum_{j=0}^{3}\left|\nabla^{j} z(x, 0)\right|^{2} d x .
\end{array}
$$

The right-hand side of (5.17) also contains the derivatives of $z$ with respect to $t$ which are not part of the initial data. Nevertheless, using (5.5), the derivatives of $z$ with respect to $t$ can be converted to $z, w$ and their derivatives with respect to $x$. The estimates for $w$ can be derivated from (5.6), (5.7), and (5.17). In conclusion, we 
have the desired energy estimates

$$
\begin{aligned}
\int_{-\infty}^{\infty}( & \left.\sum_{j=0}^{3}\left|\nabla^{j} z(x, \tau)\right|+\sum_{j=0}^{2}\left|\nabla^{j} w(z, \tau)\right|^{2}\right) d x \\
& +\int_{0}^{\tau} \int_{-\infty}^{\infty}\left(\left|\mu_{x}\right| z^{2}+\sum_{j=1}^{3}\left|\nabla^{j} z\right|^{2}+\sum_{j=0}^{2}\left|\nabla^{j} w\right|^{2}\right) d x d t \\
= & O(1) \int_{-\infty}^{\infty}\left(\sum_{j=0}^{3}\left|\frac{\partial^{j} z}{\partial x^{j}}(x, 0)\right|^{2}+\sum_{j=0}^{2}\left|\frac{\partial^{j} w}{\partial x^{j}}(x, 0)\right|^{2}\right) d x,
\end{aligned}
$$

provided that (5.13) holds. Clearly (5.13) is a consequence of the derivation of the a priori estimate (5.18). That the solution exists global in time follows from the standard local existence theory and the a priori estimate (5.18). The convergence of $(u, v)$ to $(\varphi, \psi)$ is equivalent to $\left(z_{x}, w\right) \rightarrow 0$ as $t \rightarrow \infty$. This again follows easily from (5.18) by the same arguments toward the end of the proof of Theorem 3.1. This completes the proof of the theorem. Q.E.D.

\section{Time Asymptotic Wave Patterns}

By a time-asymptotic wave pattern we mean a combination of nonlinear waves which tends to an exact solution of (1.1) and becomes noninteracting as $t \rightarrow \infty$. Consequently such wave patterns would represent the large-time states for general solutions of (1.1). From the study of Sect. 3 we see that a rarefaction wave $u$ of the equilibrium equation (1.3) together with $v=v_{*}(u)$ form a time-asymptotic wave pattern for (1.1). Sections 4 and 5 show that a smooth traveling wave of (1.1) is a time-asymptotic wave pattern if the wave speed is subcharacteristic. This is so if the wave is weak and the equilibrium speed is subcharacteristic, (4.13). In general, we have to consider traveling waves which are not smooth. By Theorem 4.1, this happens when the wave speed may equal the characteristic speed $\lambda_{1}$ or $\lambda_{2}$. Discontinuity waves for (1.1) are the same as those for the associated two conservation laws

$$
u_{t}+f(u, v)_{x}=0, \quad v_{t}+g(u, v)_{x}=0,
$$

and have been studied by Liu [5]. We now recall briefly this theory.

A discontinuity wave $\left(u_{e}, v_{e}, u_{r}, v_{r}\right)$ for (6.1) with speed $s$ satisfies the jump condition

$$
s\left(u_{r}-u_{e}\right)=f\left(u_{r}, v_{r}\right)-f\left(u_{e}, v_{e}\right), \quad s\left(v_{r}-v_{e}\right)=g\left(u_{r}, v_{r}\right)-g\left(u_{e}, v_{e}\right) .
$$

Given a fixed state $\left(u_{0}, v_{0}\right)$, let $s\left(u_{0}, v_{0}\right)$ be the set

$$
\begin{gathered}
S\left(u_{0}, v_{0}\right)=\left\{(u, v): \sigma\left(\begin{array}{c}
u-u_{0} \\
v-v_{0}
\end{array}\right)=\left(\begin{array}{l}
f(u, v)-f\left(u_{0}, v_{0}\right) \\
g(u, v)-g\left(u_{0}, v_{0}\right)
\end{array}\right)\right. \\
\text { for some scalar } \left.\sigma=\sigma\left(u_{0}, v_{0} ; u, v\right)\right\} .
\end{gathered}
$$

It turns out that, at least for a small neighborhood of $\left(u_{0}, v_{0}\right), S\left(u_{0}, v_{0}\right)$ consists of two curves $S_{1}\left(u_{0}, v_{0}\right)$ and $S_{2}\left(u_{0}, v_{0}\right)$. Along $S_{i}\left(u_{0}, v_{0}\right)$, the shock speed $\sigma$ is close to 
$\lambda_{i}, i=1,2$. The jump condition (6.2) is equivalent to

$$
s=\sigma\left(u_{e}, v_{e} ; u_{r}, v_{r}\right), \quad\left(u_{r}, v_{r}\right) \in S_{i}\left(u_{e}, v_{e}\right), \quad i=1 \text { or } 2 .
$$

In place of (4.5) for scalar conservation law (1.3), a shock wave for the system (4.13) is required to satisfy the admissibility criterion:

$$
\begin{gathered}
\sigma\left(u_{e}, v_{e} ; u_{r}, v_{r}\right) \leqq \sigma\left(u_{e}, v_{e} ; u, v\right) \text { for all }(u, v) \in S_{i}\left(u_{e}, v_{e}\right) \\
\text { between }\left(u_{e}, v_{e}\right) \text { and }\left(u_{r}, v_{r}\right) .
\end{gathered}
$$

A consequence of (4.15) is the following linearized stability criterion.

$$
\lambda_{i}\left(u_{r}, v_{r}\right) \leqq \sigma\left(u_{e}, v_{e} ; u_{r}, v_{r}\right) \leqq \lambda_{i}\left(u_{e}, v_{e}\right) .
$$

Following [6] and consistent with most physical examples, we assume that the set $S_{1}$ and $S_{2}$ are defined globally and that system (6.1) is strictly hyperbolic in the global sense. The latter means that shock speeds of one family never equal the characteristic speed of the other family.

Our purpose is to study the asymptotic state of a general solution whose initial data tend to constant states as $|x| \rightarrow \infty$. A constant state initial data for (1.1) give rise to a solution which tends to an equilibrium state at exponential rate as $t \rightarrow \infty$. Thus we assume the end states to be equilibrium. A general solution consists of many waves which interact nonlinearly and would become a simple wave pattern which is noninteracting. Consequently, our task is to find wave patterns which consist of equilibrium rarefaction waves based on the equilibrium equation (1.3), smooth traveling waves of (1.1) and discontinuity waves of (6.1). Moreover, these waves should be noninteracting, that is, a wave has a slower speed than those to the right of it. Given any two equilibrium states $\left(u_{e}, v_{*}\left(u_{e}\right)\right)$ and $\left(u_{r}, v_{*}\left(u_{r}\right)\right)$, such a wave pattern can be constructed uniquely. By (2.8) we may use the coordinate system $(u, f(u, v))$ for convenience. From (1.2) we see that the equilibrium set $\Gamma \equiv\left\{\left(u, v_{*}(u)\right)\right\}$ is a wave along which $f$ is a function of $u$ (Fig. 6.1).

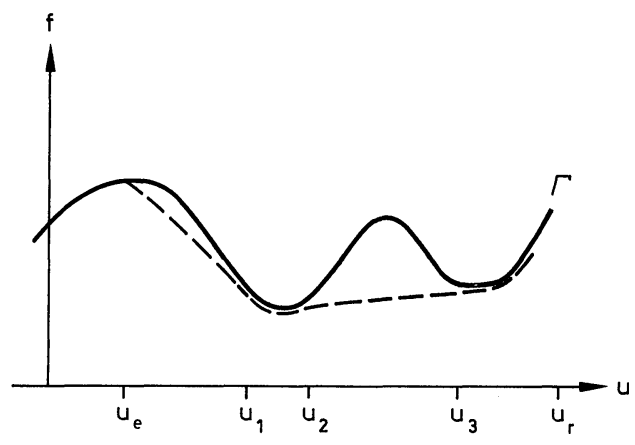

Fig. 6.1

The curve $\Gamma$ divides $(u, f)$-plane into two parts. We assume, for definiteness, that [cf. (2.8)]

$$
\frac{\partial f}{\partial v}<0
$$


so that $h>0(h<0)$ in the upper (lower) part. Along $\Gamma, f(u, v)=f\left(u, v_{*}(u)\right)=f_{*}(u)$ and so the slope of $\Gamma$ is $f_{*}^{\prime}(u)=\lambda_{*}(u)$ the equilibrium characteristic speed. Suppose that $u_{e}<u_{r}$ (the case $u_{e}>u_{r}$ is analogous). Let a string (the dotted line in Fig. 6.1) be fixed on $\Gamma$ at $u_{e}$ and $u_{r}$ and consists of straight lines lying below $\Gamma$ (between $u_{e}$ and $u_{1}$, and also between $u_{2}$ and $u_{3}$ ) and portion of $\Gamma$ (between $u_{1}$ and $u_{2}$ and also between $u_{3}$ and $\left.u_{r}\right)$. When the string coincides with $\Gamma$ it is clear that $\lambda_{*}(u)$ is increasing there and a rarefaction wave for (1.3) can be constructed. Corresponding to the straight lines in the string, a traveling wave for (1.1) can be constructed. Thus in Fig. 6.1, the asymptotic state connecting $\left(u_{e}, v_{*}\left(u_{e}\right)\right)$ and $\left(u_{r}, v_{*}\left(u_{r}\right)\right)$ consists of traveling waves $\left(u_{e}, u_{1}\right),\left(u_{r}, u_{3}\right),\left(u_{4}, u_{r}\right)$ and rarefaction waves $\left(u_{1}, u_{2}\right),\left(u_{3}, u_{4}\right)$. The construction of a rarefaction wave $(\varphi, \psi)$ is simply by setting $\varphi$ to be a rarefaction wave of the equilibrium equation (1.3) and $\psi=v_{*}(\varphi)$. Smooth traveling waves have been studied in Sect. 4 . In the present setting we note that $h<0$ along straight lines in the string. Thus we have from (4.9) and (6.5) that if the shock speed is always subcharacteristic, then a smooth traveling wave exists along each of the straight lines in the string and that $u$ is increasing along the wave. From (4.3) and the present construction, such a traveling wave moves with the same speed as the neighboring rarefaction waves and thereby is noninteracting. It remains to show that a traveling wave can still be constructed even if its speed is not subcharacteristic. Thus we consider two equilibrium states $\left(u_{-}, v_{*}\left(u_{-}\right)\right)$and $\left(u_{+}, v_{*}\left(u_{+}\right)\right)$, $u_{-}<u_{+}$, with the property that $h(u, v)<0$ along the line

$$
\begin{gathered}
L=\left\{(u, v): u_{-}<u<u_{+},-\sigma u+f(u, v)=-\sigma u_{ \pm}+f\left(u_{ \pm}, v_{ \pm}\right)=-\sigma u_{ \pm}+f_{*}\left(u_{ \pm}\right)\right\}, \\
\sigma \equiv \frac{f_{*}\left(u_{+}\right)-f_{*}\left(u_{-}\right)}{u_{+}-u_{-}} .
\end{gathered}
$$

Suppose that $\sigma$ may equal $\lambda_{1}$ or $\lambda_{2}$ along $L$. For definiteness we assume that $\sigma$ is always greater than $\lambda_{1}$ but may equal $\lambda_{2}$. Since we assume that $\left(u_{-}, u_{+}\right)$is admissible for (1.3), [cf. Fig. 6.1 and (4.5)], we have $\lambda_{*}\left(u_{+}\right) \leqq \sigma \leqq \lambda_{*}\left(u_{-}\right)$. From the basic assumption that the equilibrium speed $\lambda_{*}$ is subcharacteristic, (4.13), and that $\sigma>\lambda_{1}(u, v)$ along $L$, we have

$$
\lambda_{1}\left(u_{-}\right)<\sigma \leqq \lambda_{2}\left(u_{-}\right) .
$$

From (4.9) and (4.10) we have along $L$ that

$$
\frac{d(g(u, v)-\sigma v)}{d u}=\frac{-f_{v}}{\left(\lambda_{1}-\sigma\right)\left(\lambda_{2}-\sigma\right)} .
$$

It follows from (6.5), (6.6), and (6.8) that $g-\sigma v$ increases along $L$ near $\left(u_{-}, v_{*}\left(u_{-}\right)\right)$. As $u$ increases along $L, \sigma$ may become larger than $\lambda_{2}$ and in that case $g-\sigma v$ becomes decreasing. We make the hypothesis that $\sigma$ is mostly subcharacteristic in the sense that $\sigma-\lambda_{2}$ never becomes too large along $L$. More precisely, we assume

$$
g(u, v)-\sigma v \geqq g\left(u_{-}, v_{*}\left(u_{-}\right)\right)-\sigma v_{*}\left(u_{-}\right) \text {for }(u, v) \in L,
$$

We now construct the traveling wave connecting $\left(u_{-}, v_{*}\left(u_{-}\right)\right)$and $\left(u_{+}, v_{*}\left(u_{+}\right)\right)$first by locating states $\left(u^{1}, v^{1}\right),\left(u^{2}, v^{2}\right) \ldots$ on $L$ with the property that $g(u, v)-\sigma v$ $\geqq g\left(u^{i}, v^{i}\right)-\sigma v^{i}=g\left(u^{i+1}, v^{i+1}\right)-\sigma v^{i+1}$ along $L$ between $u^{i}$ and $u^{i+1}, i$ odd, and increasing between $u^{i}$ and $u^{i+1}, i$ even, Fig. 6.2. By (6.9) this construction is possible 


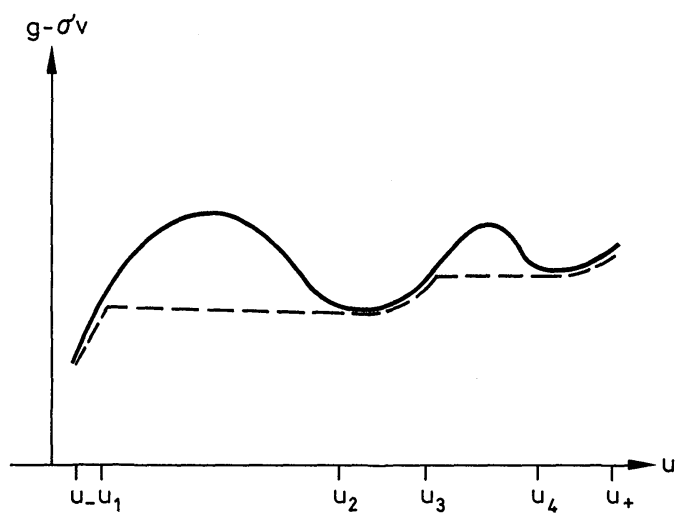

Fig. 6.2

when $g(u, v)-\sigma v$ is increasing. $\sigma$ is subcharacteristic by (6.7), and so a smooth traveling wave with speed $\sigma$ exists. Thus between $u^{i}$ and $u^{i+1}, i$ even, we have smooth traveling waves along $L$. Between $u^{i}$ and $u^{i+1}, i$ odd, we have from the construction that $\left(u^{i+1}, v^{i+1}\right) \in S_{2}\left(u^{i}, v^{i}\right)$ since the jump condition (6.6) and

$$
\sigma\left(v^{i+1}-v^{i}\right)=g\left(u^{i+1}, v^{i+1}\right)-g\left(u^{i}, v^{i}\right)
$$

are satisfied. Moreover since $g(u, v)-\sigma v \geqq g\left(u^{i}, v^{i}\right)-\sigma v^{i}$ between $u^{i}$ and $u^{i+1}$ along $L$, it can be shown easily the discontinuity waves $\left(u^{i}, v^{i} ; u^{i+1}, v^{i+1}\right), i$ odd, satisfy the admissibility condition (6.3). Thus we have the construction of a wave pattern connecting $\left(u_{-}, v_{*}\left(u_{-}\right)\right)$and $\left(u_{+}, v_{*}\left(u_{+}\right)\right)$and consisting of smooth traveling waves and admissible discontinuity waves. This completes the construction of timeasymptotic wave patterns connecting equilibrium states.

In some applications $f_{*}(u)$ is a convex function, $f_{*}^{\prime \prime}(u) \neq 0$. In this case an asymptotic wave pattern consists of either a rarefaction wave or traveling waves, Fig. 6.3 (cf. Fig. 6.1).

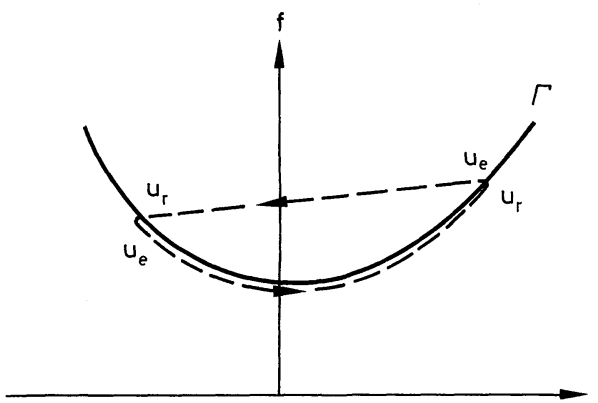

Fig. 6.3

The situation is further simplified if the characteristic value $\lambda_{i}, i=1,2$, is strictly monotone in the $r_{i}$ direction. In this case $\sigma$ is strictly monotone along the shock 


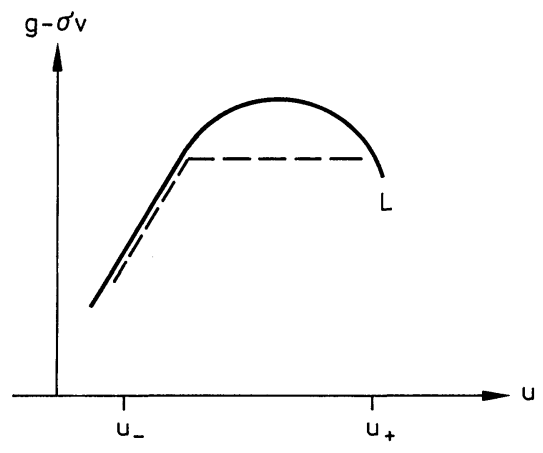

Fig. 6.4

curve $S_{i}$ and it follows that $g(u, v)-c v$ has at most one critical point, Fig. 6.4 (cf. Fig. 6.2). Consequently, a traveling wave is either smooth or consists of a smooth wave followed by a discontinuity wave.

\section{References}

1. Caflish, R., Liu, T.-P.: Nonlinear stability of shock waves for the Broadwell model (to appear)

2. Cercignani, C.: Theory and application of the Boltzman equation. Scottish Academic Press 1975

3. Dafermos, C., Nohel, J.: A nonlinear hyperbolic Volterra equation in viscoelasticity, Contribution to Analysis and Geometry, pp.87-116. Blatimore, MD.: John Hopkins University Press 1981

4. Greenberg, J., Hsiao, L.: The Riemann problem for system $u_{t}+\sigma_{x}=0$ and $\left(\sigma-f(w)_{t}\right)$ $+(\sigma-\mu f(u))=0$. Arch. Ration. Mech. Anal. 82, 87-108 (1983)

5. Liu, T.-P.: The Riemann problem for general system of conservation laws. J. Differ. Equations 18, 218-234 (1975)

6. Liu, T.-P.: Nonlinear stability of shock waves for viscous conservation laws. Mem. Am. Math. Soc. 328, No. 328 (1985)

7. Whitham, J.: Linear and nonlinear waves. New York: Wiley 1974

8. Vicenti, W., Kruger, C.: Introduction to physical gas dynamics. Melbourne: Robert E. Krieger 1982

Communicated by A. Jaffe

Received August 5, 1986 
